\title{
Color-Selective Schottky Barrier Modulation for Optoelectric Logic
}

Young Jin Choi, ${ }^{1}$ Seongchan Kim, ${ }^{2}$ Hwi Je Woo, ${ }^{2}$ Young Jae Song, ${ }^{2,3}$ Euyheon Hwang, ${ }^{2,3}$ Moon Sung Kang, ${ }^{4, *}$ and Jeong Ho Cho ${ }^{1, *}$

${ }^{1}$ Department of Chemical and Biomolecular Engineering, Yonsei University, Seoul 03722, Republic of Korea. ${ }^{2}$ SKKU Advanced Institute of Nanotechnology (SAINT), Sungkyunkwan University, Suwon 440-746, Republic of Korea.

${ }^{3}$ Department of Nano Engineering, Sungkyunkwan University, Suwon 440-746, Republic of Korea.

${ }^{4}$ Department of Chemical and Biomolecular Engineering, Institute of Emergent Materials, Sogang University, Seoul 04107, Republic of Korea.

*Corresponding authors: M. S. Kang (angms@,sogang.ac.kr) and J. H. Cho (jhcho94@yonsei.ac.kr) 


\section{Derivation of $\boldsymbol{I}-\boldsymbol{P}$ relation for photonic barristor}

We assume a first-order dependence of the photogenerated electron density $\left(\Delta n_{\mathrm{ph}}\right)$ on the light power, i.e., $\Delta n_{\mathrm{ph}}\left(P_{\mathrm{G}}\right)=k \cdot P_{\mathrm{G}}$ (where $k$ is a proportionality constant). The hole and electron densities of graphene under illumination ( $p_{\text {light }}$ and $n_{\text {light, }}$, respectively) should be

$$
\begin{aligned}
& p_{\text {light }}=p_{\text {dark }}-\Delta n_{\mathrm{ph}}=p_{\text {dark }}-k \cdot P_{\mathrm{G}}\left(\text { if } \Delta n_{\mathrm{ph}}<p_{\text {dark }}\right) \\
& n_{\text {light }}=\Delta n_{\mathrm{ph}}-p_{\text {dark }}=k \cdot P_{\mathrm{G}}-p_{\text {dark }}\left(\text { if } \Delta n_{\mathrm{ph}}>p_{\text {dark }}\right)
\end{aligned}
$$

Here, $p_{\text {dark }}\left(=4.75 \times 10^{12} \mathrm{~cm}^{-2}\right)$ is the hole density of the $p$-doped graphene under the dark condition adopted in our experiment ${ }^{1,2}$. Considering the square root dependence of the graphene work function ( $\left.W_{\text {graphene }}\right)$ on carrier density $\left(W_{\text {graphene }}=4.5+0.01165 \sqrt{p}\right.$ or $=4.5-0.01165 \sqrt{n}$, where, $p$ and $n$ are in units of $\left.10^{10} \mathrm{~cm}^{-2}\right)$, $W_{\text {graphene }}$ under illumination $\left(W_{\text {graphene }}\left(P_{\mathrm{G}}\right)\right)$ can be given as

$$
\begin{aligned}
& W_{\text {graphene }}\left(P_{\mathrm{G}}\right)=4.5+0.01165 \sqrt{p_{\text {dark }}-k \cdot P_{\mathrm{G}}}\left(\text { if } \Delta n_{\mathrm{ph}}<p_{\text {dark }}\right) \\
& W_{\text {graphene }}\left(P_{\mathrm{G}}\right)=4.5-0.01165 \sqrt{k \cdot P_{\mathrm{G}}-p_{\text {dark }}}\left(\text { if } \Delta n_{\mathrm{ph}}>p_{\text {dark }}\right)
\end{aligned}
$$

Therefore, the change in $W_{\text {graphene }}\left(P_{\mathrm{G}}\right)$ by illumination $\left(\Delta W_{\text {graphene }}\left(P_{\mathrm{G}}\right)\right)$ can be expressed as

$$
\begin{aligned}
\Delta W_{\text {graphene }}\left(P_{\mathrm{G}}\right)= & {\left[4.5+0.01165 \sqrt{p_{\text {dark }}-k \cdot P_{\mathrm{G}}}\right]-\left[4.5+0.01165 \sqrt{p_{\text {dark }}}\right] } \\
= & {\left[0.01165 \sqrt{p_{\text {dark }}-k \cdot P_{\mathrm{G}}}\right]-\left[0.01165 \sqrt{p_{\text {dark }}}\right] } \\
& \left(\text { if } \Delta n_{\mathrm{ph}}<p_{\text {dark }}\right) \\
\Delta W_{\text {graphene }}\left(P_{\mathrm{G}}\right)= & {\left[4.5-0.01165 \sqrt{k \cdot P_{\mathrm{G}}-p_{\text {dark }}}\right]-\left[4.5+0.01165 \sqrt{p_{\text {dark }}}\right] } \\
= & {\left[-0.01165 \sqrt{k \cdot P_{\mathrm{G}}-p_{\text {dark }}}\right]-\left[0.01165 \sqrt{p_{\text {dark }}}\right] } \\
& \left(\text { if } \Delta n_{\mathrm{ph}}>p_{\text {dark }}\right)
\end{aligned}
$$

Since the change in the work function of graphene ( $\left.\Delta W_{\text {graphene }}\right)$ leads to the change in the height of the Schottky barrier $\left(\Delta \varphi_{\mathrm{SB}}\right)$, the height of the Schottky barrier under illumination $\left(\varphi_{\mathrm{SB}}\left(P_{\mathrm{G}}\right)\right)$ can be given as

$$
\varphi_{\mathrm{SB}}\left(P_{\mathrm{G}}\right)=\Delta \varphi_{\mathrm{SB}}\left(P_{\mathrm{G}}\right)+\varphi_{\mathrm{SB}}(\text { dark })=\Delta W_{\text {graphene }}\left(P_{\mathrm{G}}\right)+\varphi_{\mathrm{SB}}(\text { dark })
$$

Inserting this into the saturation current $\left(I_{\mathrm{sat}}\right)$ of a standard Schottky diode equation given as

$$
I_{\text {sat }}=A A^{*} T^{2} \exp \left(-\frac{q \varphi_{S B}}{k_{\mathrm{B}} T}\right)
$$

where $A$ is the area of the junction and $A^{*}$ is the effective Richardson constant, the saturation current under illumination $\left(I_{\mathrm{sat}}\left(P_{\mathrm{G}}\right)\right)$ can be expressed as

$$
\begin{aligned}
I_{\text {sat }}\left(P_{\mathrm{G}}\right)= & I_{\text {sat }}(\text { dark })\left\{\exp \left(-\frac{q\left[0.01165 \sqrt{p_{\text {dark }}-k \cdot P_{\mathrm{G}}}\right]}{k_{\mathrm{B}} T}+\frac{q\left[0.01165 \sqrt{p_{\text {dark }}}\right]}{k_{\mathrm{B}} T}\right)\right\} \\
& \left(\text { if } \Delta n_{\mathrm{ph}}<p_{\text {dark }}\right) \\
I_{\text {sat }}\left(P_{\mathrm{G}}\right)= & I_{\text {sat }}(\text { dark })\left\{\exp \left(+\frac{q\left[0.01165 \sqrt{k \cdot P_{\mathrm{G}}-p_{\text {dark }}}\right]}{k_{\mathrm{B}} T}+\frac{q\left[0.01165 \sqrt{p_{\text {dark }}}\right]}{k_{\mathrm{B}} T}\right)\right\} \\
& \left(\text { if } \Delta n_{\mathrm{ph}}>p_{\text {dark }}\right)
\end{aligned}
$$


where,

$$
I_{\text {sat }}(\text { dark })=A A^{*} T^{2} \exp \left(-\frac{q \varphi_{S B}(\text { dark })}{k_{\mathrm{B}} T}\right)
$$

Finally, the current-illumination power $\left(I_{\mathrm{D}}-P_{\mathrm{G}}\right)$ equation for the photonic barristor is given as

$$
\begin{gathered}
I_{\mathrm{D}}=I_{\text {sat }}(\text { dark })\left\{\exp \left(-\frac{q\left[0.01165 \sqrt{p_{\text {dark }}-k \cdot P_{\mathrm{G}}}\right]}{k_{\mathrm{B}} T}+\frac{q\left[0.01165 \sqrt{p_{\text {dark }}}\right]}{k_{\mathrm{B}} T}\right)\right\}\left\{\exp \left(\frac{q V_{\mathrm{D}}}{k_{\mathrm{B}} T}\right)-1\right\} \\
I_{\mathrm{D}}=I_{\text {sat }}(\text { dark })\left\{\exp \left(+\frac{q\left[0.01165 \sqrt{k_{\mathrm{ph}} P_{\mathrm{G}}-p_{\text {dark }}}\right]}{k_{\mathrm{B}} T}+\frac{q\left[0.01165 \sqrt{p_{\text {dark }}}\right]}{k_{\mathrm{B}} T}\right)\right\}\left\{\exp \left(\frac{q V_{\mathrm{D}}}{k_{\mathrm{B}} T}\right)-1\right\} \\
\left(\text { if } \Delta n_{\mathrm{ph}}>p_{\text {dark }}\right)
\end{gathered}
$$




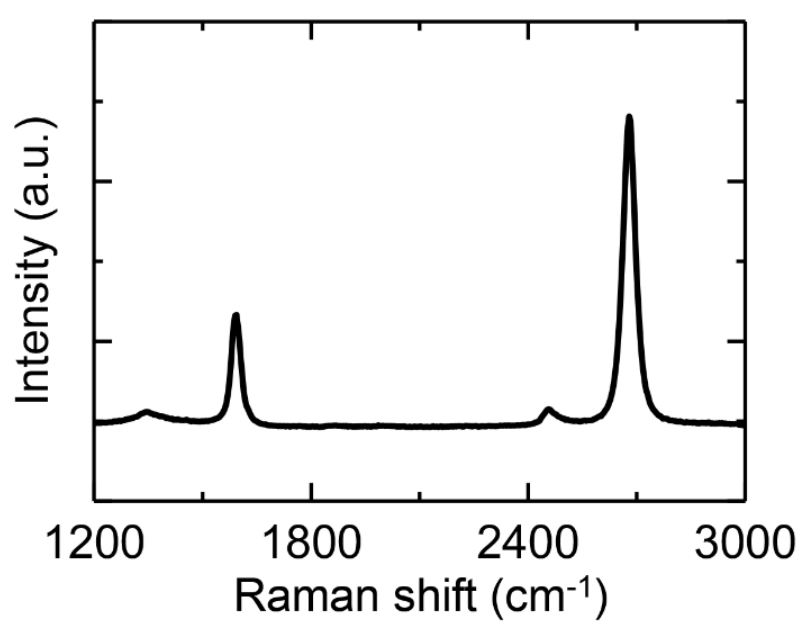

Figure S1. Raman spectrum of CVD-grown monolayer graphene.

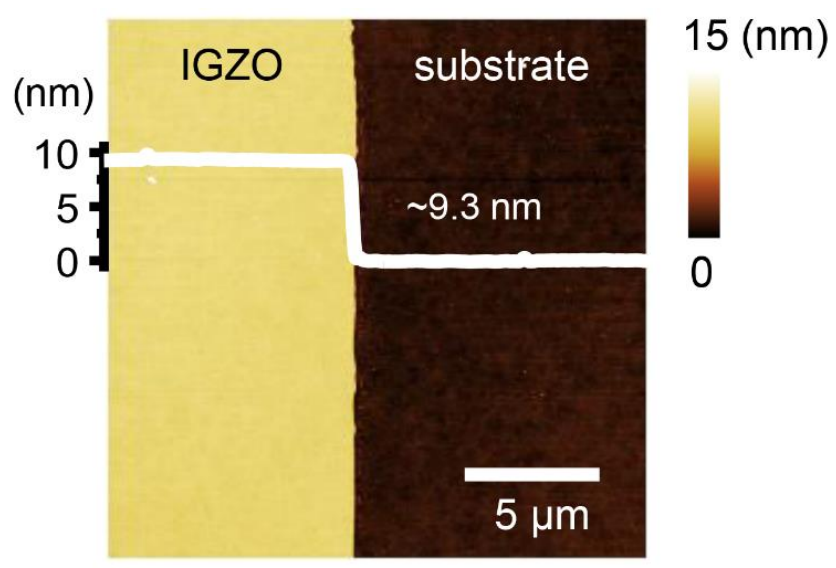

Figure S2. Surface morphology and height profile of the patterned IGZO.

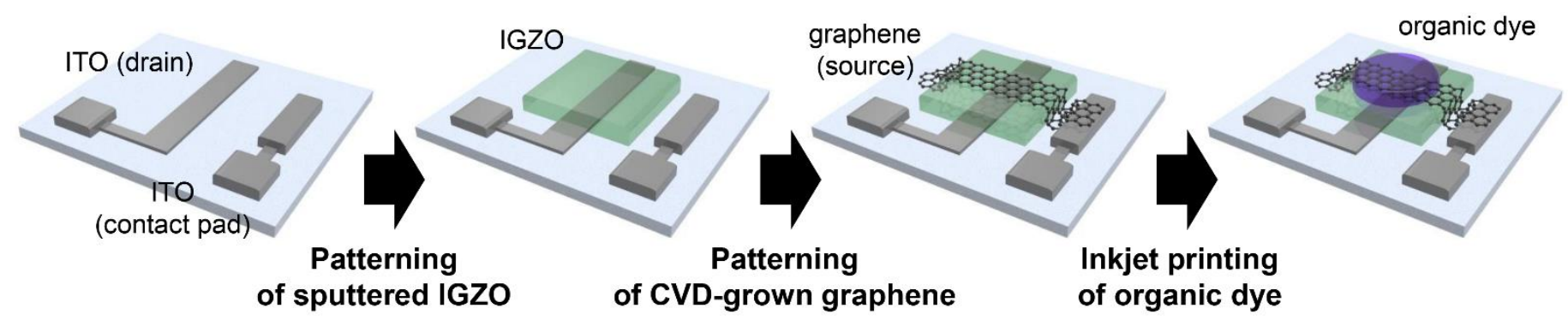

Figure S3. Schematic fabrication procedure of photonic barristor. 


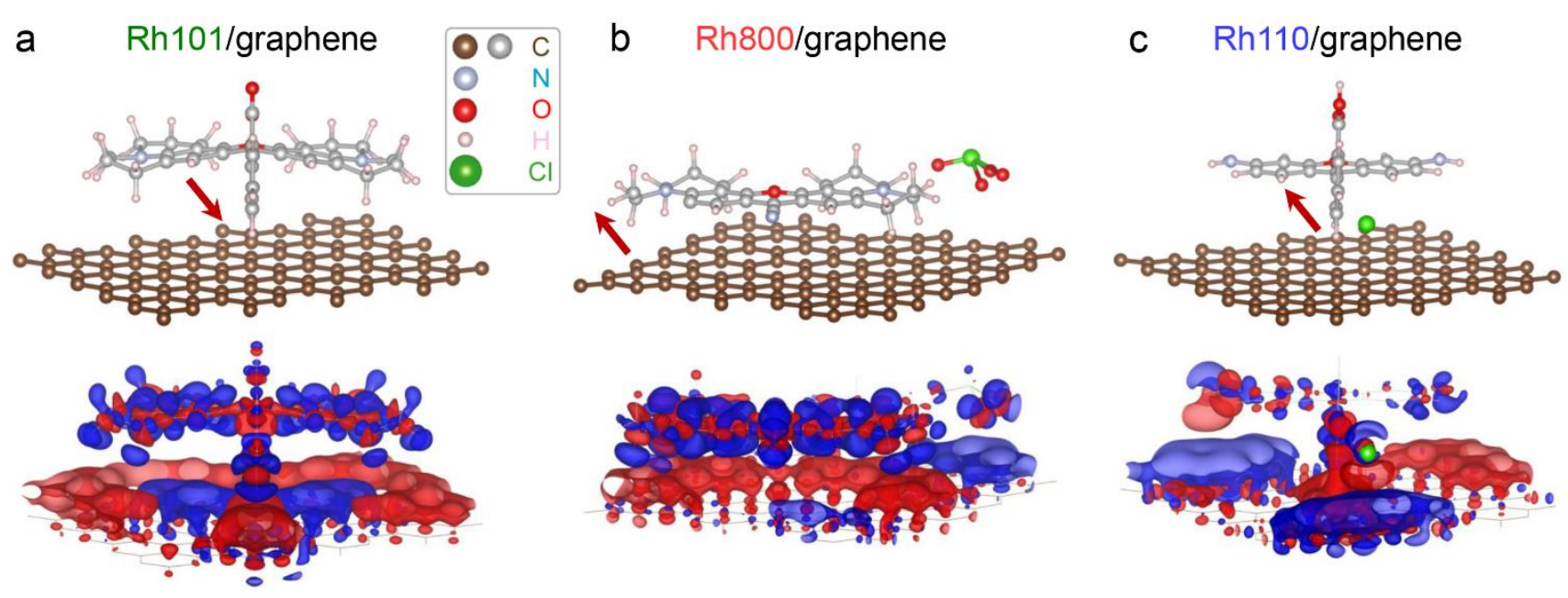

Figure S4. Optimized interface structures and electron density difference isosurfaces of organic dye/graphene heterostructures: (a) Rh101, (b) Rh800, and (c) Rh110.
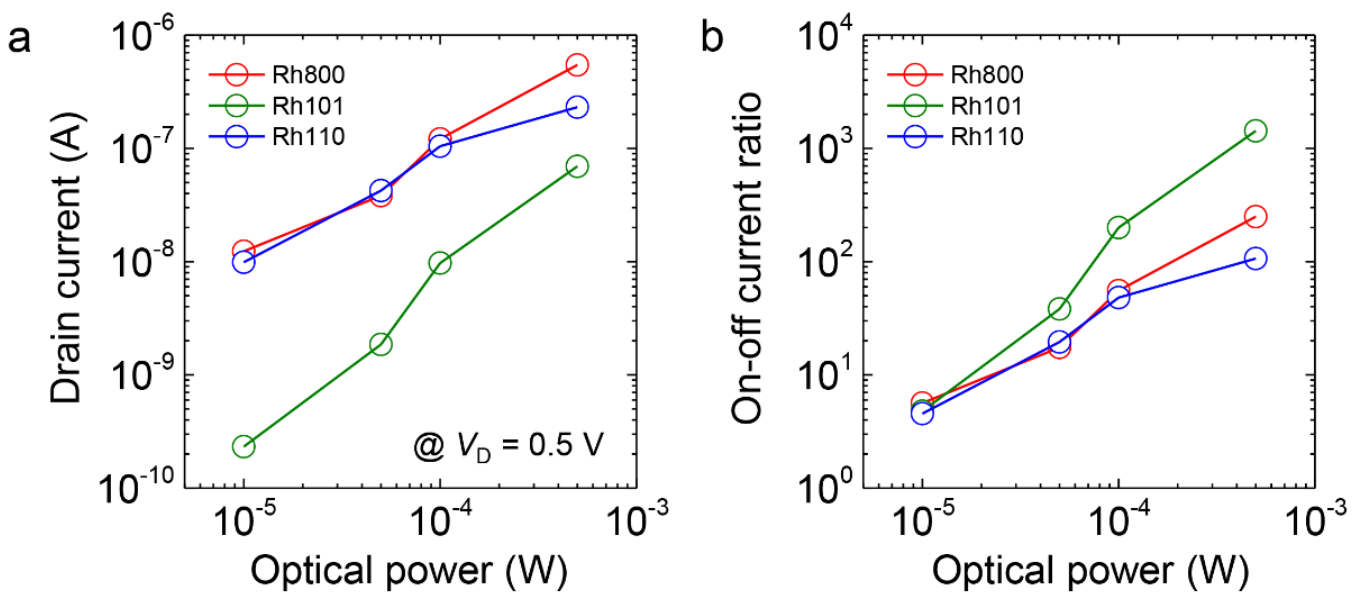

Figure S5. (a) Drain current and (b) on-off current ratio of photonic barristors with Rh800, Rh101, and Rh110 under light illumination with various optical powers.

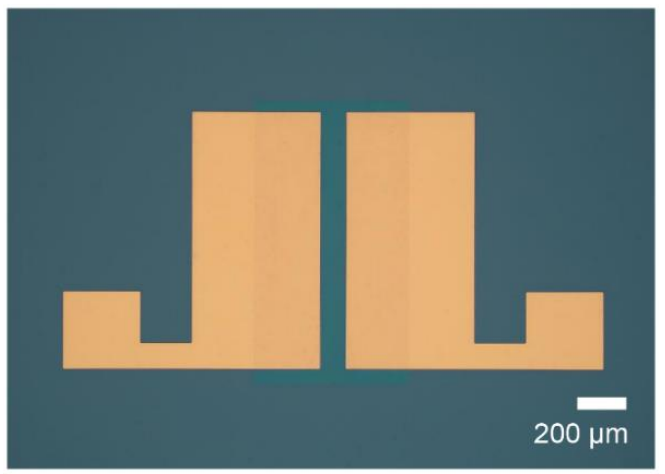

Figure S6. Optical microscopy image of lateral transistor based on Rh101-doped graphene channel. 


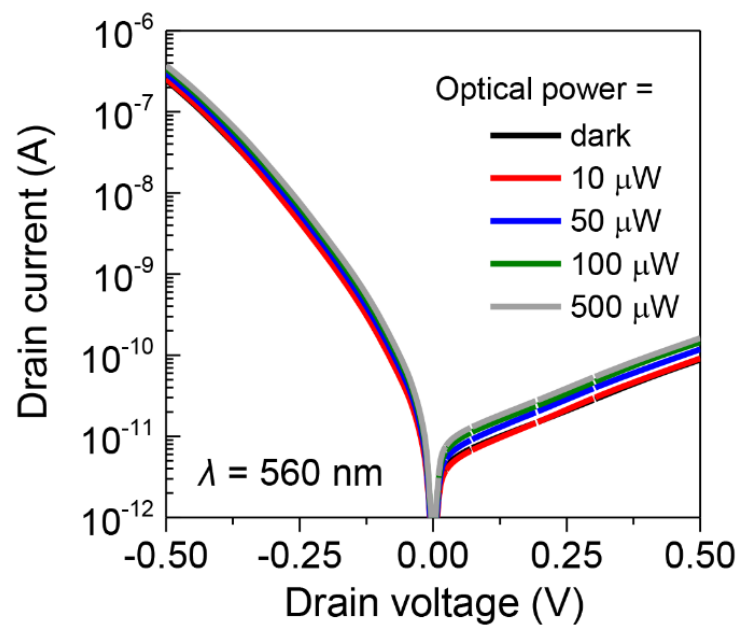

Figure S7. Current-voltage characteristics of load photonic barristor under light illumination with various optical powers. No short circuit current and open circuit potential were observed under illumination from our devices because no photo generation of extra charge carriers could take place within the transparent source electrode, channel, and drain electrode.

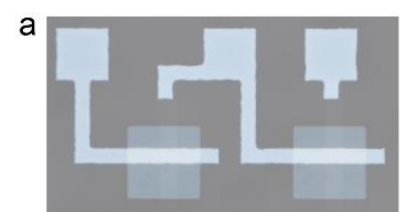

input $=0$

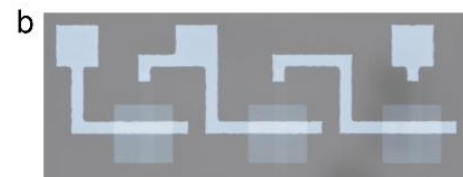

input $=(0,0)$

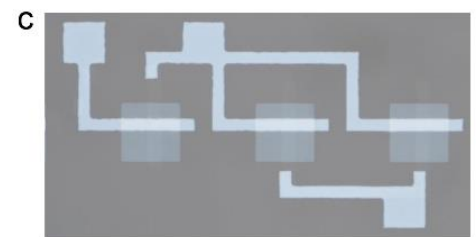

input $=(0,0)$

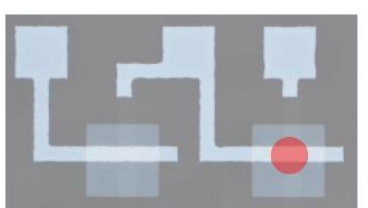

input $=1$

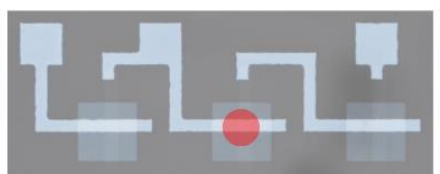

$(1,0)$

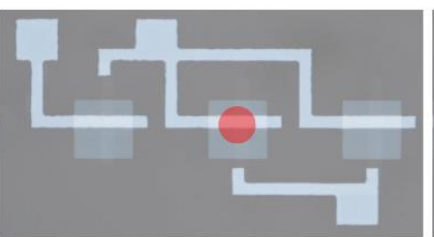

$(1,0)$

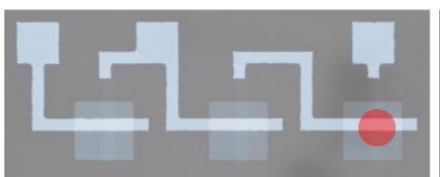

$(0,1)$

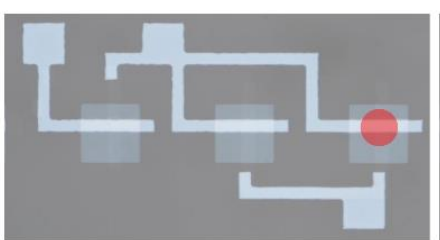

$(0,1)$

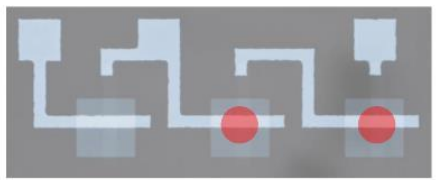

$(1,1)$

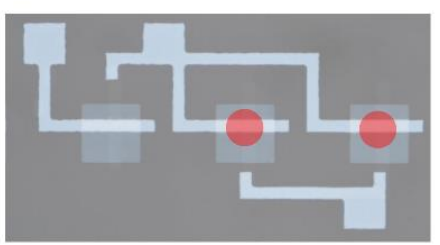

$(1,1)$

Figure S8. Schematics of irradiation conditions of optoelectric logics in different optical input states: (a) NOT, (b) NAND, and (c) NOR logics. 


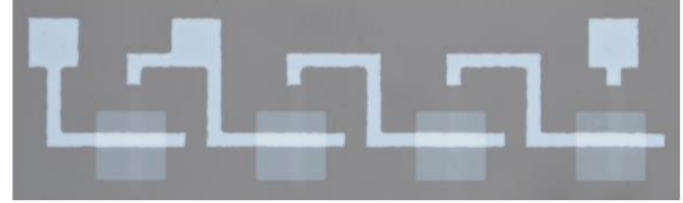

input $=(0,0,0)$

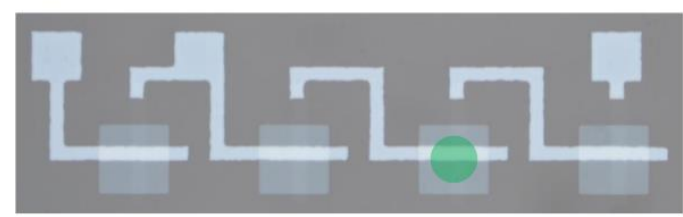

$(0,1,0)$

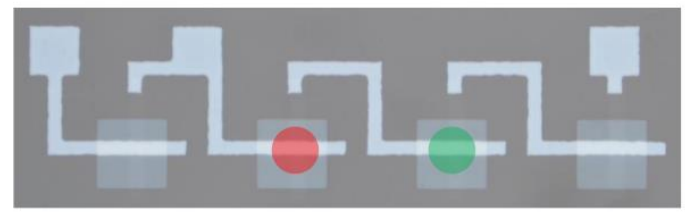

$(1,1,0)$

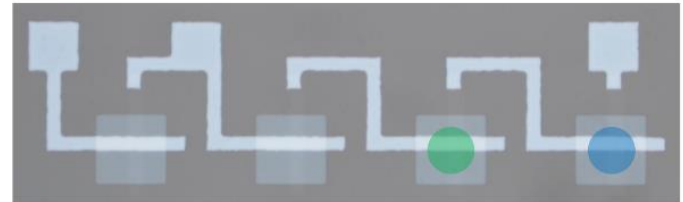

$(0,1,1)$

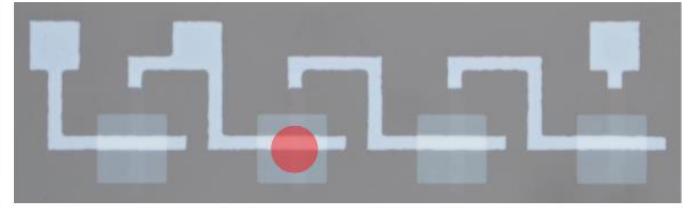

$(1,0,0)$

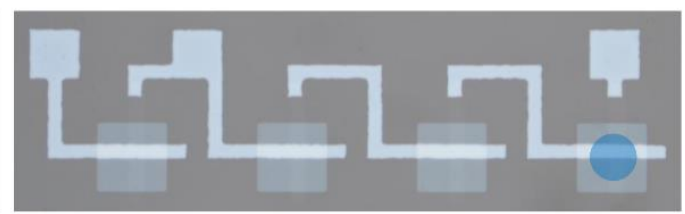

$(0,0,1)$

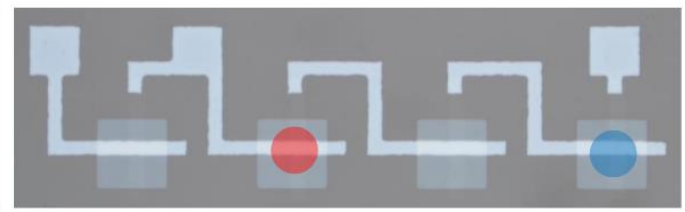

$(1,0,1)$

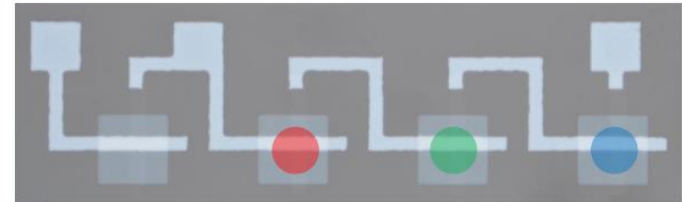

$(1,1,1)$

Figure S9. Schematics of irradiation conditions of three-input RGB NAND optoelectric logic in different optical input states.

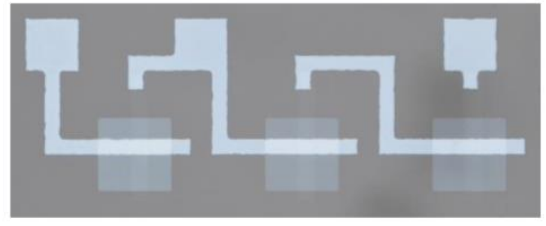

$$
\text { input }=(0,0)
$$

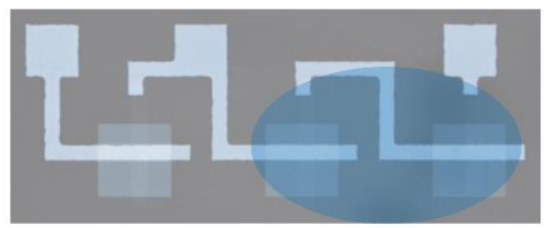

$(0,1)$

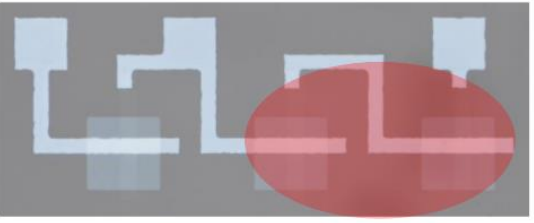

$(1,0)$

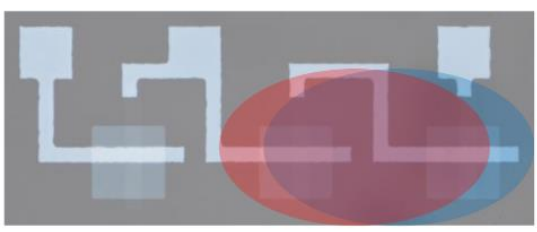

$(1,1)$

Figure S10. Schematics of irradiation conditions of two-input selective RB NAND optoelectric logic in different optical input states. 


\section{REFERENCES}

1. Wang, Q. H.; Jin, Z.; Kim, K. K.; Hilmer, A. J.; Paulus, G. L. C.; Shih, C.-J.; Ham, M.-H.; SanchezYamagishi, J. D.; Watanabe, K.; Taniguchi, T.; Kong, J.; Jarillo-Herrero, P.; Strano, M. S.

Understanding and Controlling the Substrate Effect on Graphene Electron-Transfer Chemistry via Reactivity Imprint Lithography. Nat. Chem. 2012, 4, 724-732.

2. Joshi, P.; Romero, H. E.; Neal, A. T.; Toutam, V. K.; Tadigadapa, S. A. Intrinsic Doping and Gate Hysteresis in Graphene Field Effect Devices Fabricated on $\mathrm{SiO}_{2}$ Substrates. J. Phys.: Condens. Matter 2010, 22, 334214. 